\title{
Características produtivas e morfológicas de frutos de tomate em resposta a sistemas de raleio
}

\author{
Tiago Aparecido PARCA ${ }^{1}$, Everton Geraldo de MORAIS ${ }^{2 *}$, Henrique José Guimarães Moreira MALUF², \\ Luciano Donizete GONÇALVES ${ }^{1}$, Diorge Maykon de OLIVEIRA ${ }^{1}$, Willian Douglas DUARTE ${ }^{1}$
}
${ }^{1}$ Departamento de Ciências Agrárias, Instituto Federal de Educação, Ciência e Tecnologia de Minas Gerais, Bambuí, MG, Brasil. ${ }^{2}$ Departamento de Ciência do Solo, Universidade Federal de Lavras, Lavras, MG, Brasil. E-mail: evertonmoraislp@yahoo.com.br

Recebido em outubro/2018; Aceito em abril/2019.

\begin{abstract}
RESUMO: O objetivo do presente trabalho foi avaliar o efeito de técnicas de raleio na produção e em características morfológicas de frutos de tomate. $\mathrm{O}$ tomateiro foi submetido a seis diferentes técnicas de raleio de frutos, com condução de diferentes números de racemos por planta. Ao final do experimento, após a colheita, foi avaliado o diâmetro médio longitudinal (DLM) e transversal (DTM) dos frutos, número de frutos por planta (NF), peso médio de frutos (PMF), produção total por planta (Prod T), produção de frutos grande (Prod G), médio (Prod M) e pequeno (Prod P), e a frequência relativa de cada classe de diâmetro. A técnica de raleio que consistiu na condução de plantas com 7 racemos, com 6 frutos por racemo até o $4^{\circ}$ racemo e acima deste, 4 frutos por racemo (R3) aumentou a Prod G, a frequência relativa da classe grande, a Prod T, DTM, DLM e PMF. Técnicas de raleio que resultaram em maior NF reduziram a qualidade morfológica dos frutos, bem como a condução de plantas com menos de sete racemos reduziu a produção de tomates. A adoção da técnica R3 no tomateiro reuni alta produção de frutos por planta com aumento da qualidade morfológica do fruto.

Palavras-chave: Solanum lycopersicum L.; desbaste de frutos; qualidade de tomates; classes de diâmetro de frutos; relação fonte:dreno.
\end{abstract}

\section{Productive and morphological features of tomato fruits in response to thinning systems}

\begin{abstract}
The aim of this work was to evaluate the effect of thinning techniques on the production and morphological features of tomato fruits. Tomato plants were submitted to six different thinning practices, with different numbers of racemes per plant. At the end of the experiment, the mean longitudinal diameter (MLD) and transverse diameter (MTD) of fruits, number of fruits per plant (NF), average fruit weight (AFW), total production per plant (T Prod), production of fruits large (Prod L), middle (Prod M) and small (Prod S), and the relative frequency of each diameter class. The thinning technique, which consisted in the conduction of plants with 7 racemes, with 6 fruits per raceme up to the $4^{\text {th }}$ raceme and above this, 4 fruits per raceme (R3) increased the Prod L, the relative frequency of the large class, T Prod, MLD, MTD, and AFW. Thinning techniques that provide greater NF reduced the morphological quality of fruits, as well as the conduction of plants with less than seven racemes decreased tomato production. Adoption of the R3 technique in the tomato plants bring together high fruit production with an increase in the fruit morphological quality.
\end{abstract}

Keywords: Solanum lycopersicum L.; fruit pruning; tomato quality; fruit diameter classes; source:sink ratio.

\section{INTRODUÇÃO}

A cultura do tomateiro é de grande importância econômica, em que diversos fatores, como condições edafoclimáticas e manejo cultural, influenciam a produção e a qualidade de seus frutos, bem como a rentabilidade dos produtores (BECKLES, 2012; MATOS et al., 2012; SCHMIDT et al., 2018; SILVA et al., 2017). Uma das maneiras de agregar valor a essa cultura no mercado é produzir frutos de maior tamanho. Tomates com maiores dimensões propiciam incremento do peso médio, favorece a comercialização para o consumo in natura e alcança preços mais elevados (GUIMARÃES et al., 2008). Esse tamanho magnificado dos frutos para a comercialização de tomate do grupo Santa Cruz é baseado, especialmente, pelo aumento no diâmetro transversal do fruto, até $100 \mathrm{~mm}$, valorlimite estabelecido pelo mercado (MONTE et al., 2013).

Desse modo, práticas que favoreçam o crescimento e melhoria das características morfológicas do fruto devem ser almejadas, como o raleio de frutos, cuja técnica atende tal objetivo, sendo de fácil execução (SOWLEY; DAMBA, 2013). O raleio consiste na retirada do excesso de frutos de menor potencial comercial, o que diminui a competição entre fotoassimilados e favorece a qualidade dos frutos remanescentes (ABDEL-RAZZAK et al., 2016; MAX et al., 2016; PENGFEI et al., 2017; RODRÍGUEZ-MENDOZA et al., 2015; SHIRAHIGE et al., 2010). O raleio contribui também para maior penetração da radiação solar no dossel da planta, o que aumenta a assimilação líquida de fotoassimilados $\mathrm{e}$, consequentemente, melhora a qualidade dos frutos de tomate (GUIMARÃES et al., 2008; MATSUDA et al., 2011; MAX et al., 2016)

O tomateiro pode ser dividido em unidades fisiológicas, caracterizadas como fonte e dreno. As folhas são consideradas fonte de fotoassimilados, sintetizados a partir da fotossíntese, enquanto os frutos são órgãos de reserva da planta, definidos como dreno desses compostos orgânicos (ENGELS et al., 2012). Dessa forma, grande parte dos fotoassimilados 
sintetizados nas folhas são preferencialmente translocados para os frutos (DUARTE; PEIL, 2010; MATSUDA et al., 2011; SHIRAHIGE et al., 2010). O raleio influência essa relação fonte/dreno do tomateiro, o que modifica a qualidade e as características morfológicas dos frutos remanescentes desse manejo (BECKLES, 2012; MABOKO et al., 2017; MATSUDA et al., 2011; SHIRAHIGE et al., 2010; SOWLEY; DAMBA, 2013). Assim, busca-se um equilíbrio entre o aumento da produção de frutos grandes e a manutenção da produtividade, cujo equilíbrio pode ser alcançado pelo tipo certo de raleio empregado (MAX et al., 2016; PENGFEI et al., 2017).

O raleio pode aumentar o tamanho dos frutos de tomate em até 20\% (SHIRAHIGE et al., 2010), contudo, poucos trabalhos relacionam esse efeito da produção do tomate em classes de diâmetro, avaliando somente a produção total do tomateiro (GUIMARÃES et al., 2008). Nesse aspecto, há carência de trabalhos que avaliam a quantidade de frutos remanescentes e sua qualidade morfológica, de forma a estabelecer técnicas de raleio que conciliem a manutenção da produtividade com aumento no tamanho dos frutos (AZEVEDO et al., 2010; BECKLES, 2012; HESAMI et al., 2012; MABOKO et al., 2011; MATSUDA et al., 2011; PENGFEI et al., 2017; SOWLEY; DAMBA, 2013). Essas avaliações são importantes para estabelecer uma prática de raleio adequada para o tomateiro.

Embora essa prática seja recomendada, muitas vezes não é adotada por receio em queda de produção de tomates. Desse modo, a principal hipótese desse estudo é que pelo menos uma técnica de raleio aumente o tamanho e a classe de diâmetro de frutos, sem reduzir a produção total do tomateiro. Para isso, o objetivo do estudo foi avaliar o peso dos frutos, seus diâmetros transversal e longitudinal, o número de tomates produzidos por planta, a produção total e as classes comerciais de diâmetro de frutos submetidos a diferentes técnicas de condução e raleio.

\section{MATERIAL E MÉTODOS}

O estudo foi conduzido no Instituto Federal Minas Gerais, campus Bambuí, localizado nas coordenadas $20^{\circ} 02^{\prime} 17^{\prime \prime} \mathrm{S}$ e 4600'32" O, município de Bambuí, Centro Oeste do estado de Minas Gerais. O clima da região é subtropical de inverno seco, segundo a classificação de Koppen. O local do estudo apresenta altitude de $706 \mathrm{~m}$, temperatura média anual de 21,3 ${ }^{\circ} \mathrm{C}$ e precipitação de $1.369 \mathrm{~mm}$.

O experimento foi constituído por seis tratamentos, referente a diferentes técnicas de condução e raleio nos frutos de tomate, sendo: plantas conduzidas sem raleio (R1); plantas conduzidas com 7 racemos, sendo que até o $4^{\circ}$ racemo sem raleio e acima deste, mantendo 4 frutos por racemo (R2); plantas conduzidas com 7 racemos, com 6 frutos até o $4^{\circ}$ racemo e acima deste, com 4 frutos (R3); plantas conduzidas com 5 racemos, sem raleio dos frutos (R4); plantas conduzidas com 5 racemos, sendo que até o $4^{\circ}$ racemo sem raleio e acima deste, mantendo 4 frutos (R5); plantas conduzidas com 5 racemos, com 6 frutos até o $4^{\circ}$ racemo, e acima deste com 4 frutos (R6) (Figura 1). Utilizou-se delineamento em blocos casualizados, com quatro repetições. A parcela experimental foi constituída por cinco plantas de tomate, em que apenas as três centrais foram avaliadas, o que definiu a parcela útil.

As plantas de tomate foram cultivadas em estufa com abertura lateral, sob espaçamento de 1,0 x 0,6 m, conduzidas com uma haste por planta e tutoradas verticalmente por meio de fitilhos ligados à estrutura fixa da estufa. A irrigação foi realizada por gotejamento, com mangueiras de diâmetro de 1/2 polegada, gotejadores espaçados de $0,6 \mathrm{~m}$ e vazão por gotejador de $1,5 \mathrm{~L} \mathrm{hora}^{-1}$.

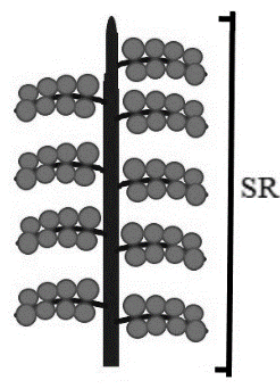

R1

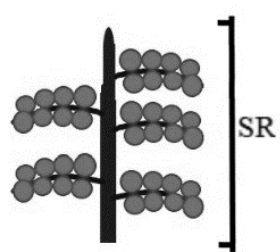

R4

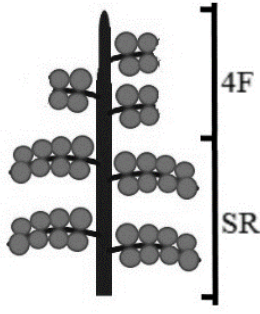

R2

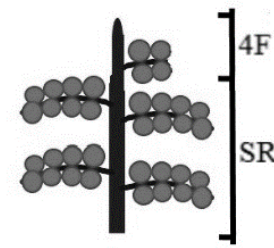

R5

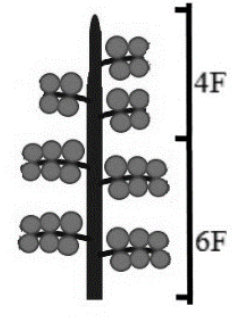

R3

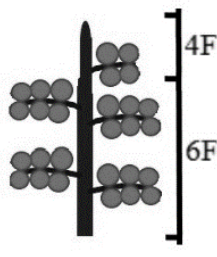

R6
Figura 1. Esquema das técnicas de raleio estudadas na planta de tomate (R1, R2, R3, R4, R5 e R6). SR: frutos sem raleio no racemo; $4 \mathrm{~F}$ : quatro frutos por racemo; $6 \mathrm{~F}$ : seis frutos por racemo.

Figure 1. Scheme of the thinning techniques studied in tomato plant (R1, R2, R3, R4, R5, and R6). SR: non-fruit thinning in raceme; 4F: four fruits in raceme; $6 \mathrm{~F}$ : six fruits in raceme.

O solo cultivado com o tomateiro foi classificado como Latossolo Vermelho distroférrico típico de textura argilosa, com os seguintes atributos químicos da camada de 0 a $20 \mathrm{~cm}$ : $\mathrm{pH}$ em $\mathrm{H}_{2} \mathrm{O}$ de 7,3; P disponível (Mehlich-1) de $965 \mathrm{mg} \mathrm{dm}^{-3}$; $\mathrm{K}$ disponível de $540 \mathrm{mg} \mathrm{dm}{ }^{-3} ; \mathrm{Ca}^{2+}, \mathrm{Mg}^{2+} \mathrm{e} \mathrm{Al}^{3+}$ trocáveis de $11,2,1,3$ e $0,0 \mathrm{cmol}_{\mathrm{c}} \mathrm{dm}^{-3}$, respectivamente; CTC a pH 7 de $15,2 \mathrm{cmol}_{\mathrm{c}} \mathrm{dm}^{-3}$; saturação por bases de $91,7 \%$; matéria orgânica de 26,8 $\mathrm{g} \mathrm{kg}^{-1}$; P-rem de 28,0 mg L${ }^{-1} ; \mathrm{Zn}, \mathrm{Mn}, \mathrm{Cu}, \mathrm{B}$ e $\mathrm{S}$ disponíveis de 35,1, 94,7, 8,5, 1,1 e 63,4 $\mathrm{mg} \mathrm{dm}^{-3}$, respectivamente. $\mathrm{Na}$ camada de 20 a $40 \mathrm{~cm}$, verificou-se: $\mathrm{pH}$ em $\mathrm{H}_{2} \mathrm{O}$ de 7,4; $\mathrm{P}$ disponível (Mehlich-1) de $1.263 \mathrm{mg} \mathrm{dm}^{-3} ; \mathrm{K}$ disponível de $550 \mathrm{mg} \mathrm{dm}{ }^{-3} ; \mathrm{Ca}^{2+}, \mathrm{Mg}^{2+}$ e $\mathrm{Al}^{3+}$ de $11,5,1,4 \mathrm{e}$

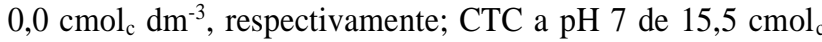
$\mathrm{dm}^{-3}$; saturação por bases de $91,9 \%$; matéria orgânica de 26,1 $\mathrm{g} \mathrm{kg}^{-1}$; e P-rem de $26,3 \mathrm{mg} \mathrm{L}^{-1}$. Dessa forma, não houve necessidade de correção da acidez do solo cultivado e, a adubação foi realizada de acordo com as recomendações propostas por Filgueira et al. (1999) para a cultura do tomateiro.

As mudas de tomate, híbrido Cienaga grupo Santa Cruz, foram produzidas em bandejas de poliestireno expandido com 128 células, utilizando substrato comercial a base de fibra de coco. O transplante das mudas para a área de cultivo foi realizado quando as plantas apresentaram o estádio de quatro folhas definitivas.

Durante o cultivo não houve necessidade de controle fitossanitário, uma vez que não se observou incidência de pragas e, ou de doenças a níveis que justificassem o controle. A desbrota dos ramos ladrões das plantas e as capinas foram realizadas manualmente ao longo do cultivo. A prática de amontoa foi realizada após o transplante, quando as plantas apresentavam $30 \mathrm{~cm}$ de altura. As diferentes técnicas de raleio empregadas no estudo foram realizadas logo após o início da 
frutificação. Por fim, a colheita dos frutos de tomate se iniciou aos 90 dias do transplante, realizada semanalmente até o fim do ciclo da cultura, de acordo com a maturação dos frutos.

Após a colheita, os frutos de cada planta foram contados, a fim de determinar o número de frutos por planta (NF) e mensurados os diâmetros transversal (DTM) e longitudinal (DLM) com uso de paquímetro digital. Com o diâmetro transversal, os frutos foram separados em classes, de acordo com a classificação oficial disposta na norma de identidade, qualidade, acondicionamento, embalagem e apresentação do tomate do Ministério da Agricultura, Pecuária e Abastecimento (MAPA, 1995). Esta normativa classifica o fruto do tomateiro, como Grande (diâmetro transversal maior que $60 \mathrm{~mm}$ ), Médio (maior que $50 \mathrm{~mm}$ e menor ou igual a 60 $\mathrm{mm}$ ) e Pequeno (maior que $40 \mathrm{~mm}$ e menor ou igual a $50 \mathrm{~mm}$ ). Após a separação por classe de diâmetro, os frutos foram pesados com o uso de balança analítica digital, com precisão de duas casas decimais, a fim de determinar o peso médio dos frutos (PMF), dividindo a produção total por planta pela quantidade de frutos produzido. Além disso, foi determinado a frequência relativa de cada classe de diâmetro (FCD), por meio da relação entre produção de cada classe e produção total por planta (Equação 1).

$$
\text { FCD }(\%)=(P C D \div \operatorname{Prod} T) \times 100
$$

em que: PCD é a produção de frutos de tomate em determinada classe de diâmetro, em kg planta ${ }^{-1}$; e Prod T é a produção total de tomate, em kg planta ${ }^{-1}$.

Os dados coletados foram submetidos à análise de variância e quando significativo pelo teste $F(p<0,05)$, as médias de cada tratamento foram agrupadas pelo teste ScottKnott $(\mathrm{p}<0,05)$, utilizando o pacote Exp.Des.pt 1.1.2 (FERREIRA et al., 2013), instalado no programa $R$ (R CORE TEAM, 2018). A análise de componentes principais também foi realizada, a fim de verificar a relação múltipla e linear entre as variáveis avaliadas e os tratamentos, por meio do pacote vegan 2.4-3 (OKSANEN, 2017) do programa $R$ (R CORE TEAM, 2018).

\section{RESULTADOS}

A condução das plantas, com 6 frutos por racemo até o $4^{\circ}$ racemo e acima deste, mantendo 4 frutos por racemo, seja com condução de 7 racemos por planta (R3) ou 5 racemos por planta (R6), aumentou o diâmetro transversal médio (DTM), longitudinal (DLM) e o peso médio dos frutos (PMF) em torno de $10,1,9,6$, e $31,7 \%$, respectivamente, em relação ao cultivo sem raleio (R1) (Figura 2). Entretanto, as técnicas de raleio R3 e R6 reduziram em média o número de frutos por planta (NF) em $31,9 \%$. A técnica de condução com 5 racemos, sem raleio até o $4^{\circ}$ racemo e acima deste, mantendo 4 frutos por racemo (R5) incrementou o DTM e o DLM, mas não alterou o PMF quando comparado ao R1.

As técnicas de raleio R1 e R2 contribuíram para o aumento do NF, apresentando na média das duas técnicas 33 frutos por planta (Figura 2), o que está relacionado ao próprio manejo que favorece maior quantidade de frutos em relação aos demais tratamentos. De acordo com os atributos avaliados, foi observado que o aumento do PMF pela técnica R6 está relacionado à redução do NF. Esse efeito também foi verificado no uso da técnica R3.
Quando adotou R1, a produção de frutos da classe de diâmetro grande (Prod G) foi reduzida. Essa redução também foi observada em plantas conduzidas com 5 racemos (R4) (Figura 3A). As técnicas de raleio R2, R3, R5 e R6 aumentaram a Prod $\mathrm{G}$ em 0,5 vezes em relação à média de $\mathrm{R} 1$ e R4. A produção de frutos médio (Prod M) e pequeno (Prod P) foi maior quando se utilizou os manejos R1, R2 e R4, com aumentos na ordem de 0,9 e 3,8 vezes, respectivamente para Prod $\mathrm{M}$ e Prod $\mathrm{P}$ em relação à média de R3, R5 e R6. Com o somatório da produção em classes de diâmetro, verificou-se que o cultivo de plantas com 5 racemos (R4, R5 e R6) diminuiu a produção total de frutos por planta (Prod $\mathrm{T}$ ), aproximadamente em $0,9 \mathrm{~kg}$ planta $^{-1}$, em relação as demais técnicas estudadas.
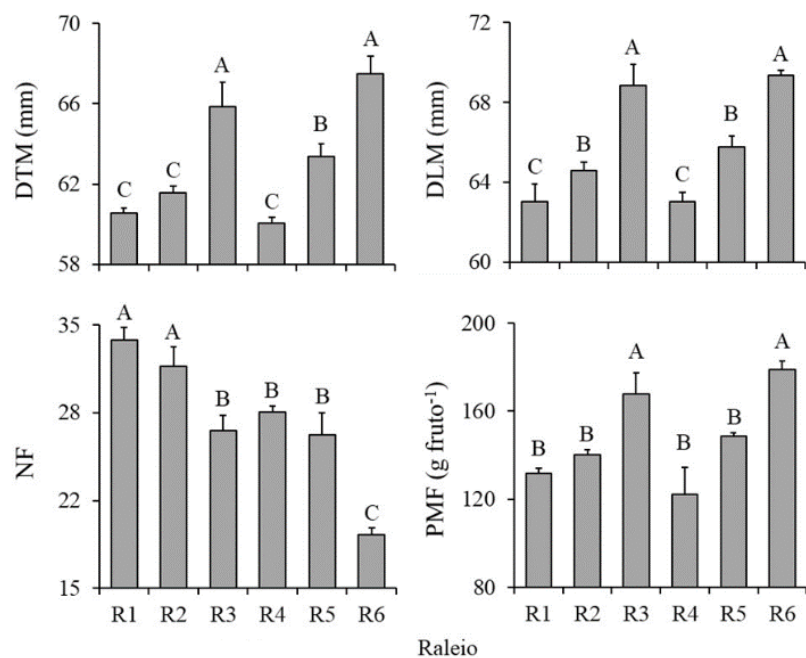

Figura 2. Diâmetro transversal médio dos frutos (DTM), longitudinal (DLM), número de frutos por planta (NF), peso médio dos frutos (PMF) de tomate em função de diferentes técnicas de raleio (R1, R2, R3, R4, R5 e R6). Barras seguidas de letras iguais, se agrupam pelo teste Scott-Knott $(\mathrm{p}<0,05)$.

Figure 2. Mean transversal diameter of fruits (MTD), longitudinal (MLD), number of fruits per plant (NF), average weight of fruits (AWF) as a function of different thinning techniques (R1, R2, R3 R4, R5, and R6). Bars followed by equal letters are grouped by the ScottKnot test $(\mathrm{p}<0.05)$
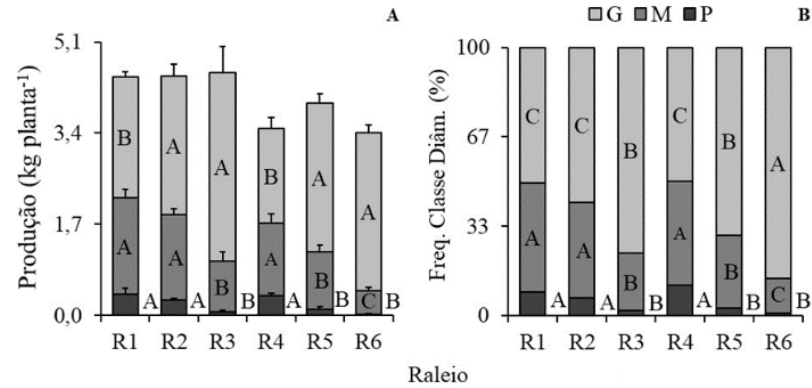

Figura 3. Produção de tomate por planta de acordo com a classe de diâmetro (A) e frequência relativa de cada classe de diâmetro de acordo com a produção total (Freq. Classe. Diâm.) (B) em função de diferentes técnicas de raleio (R1, R2, R3, R4, R5 e R6). G: Classe de diâmetro grande; M: Classe de diâmetro médio; P: classe de diâmetro pequeno. Barras seguidas de letras iguais na mesma classe de diâmetro, se agrupam pelo teste Scott-Knott $(\mathrm{p}<0,05)$.

Figure 3. Production of tomato per plant according to diameter class (A) and relative frequency of each diameter class according to total production (Freq. Classe Diam.) (B) as a function of different thinning techniques (R1, R2, R3, R4, R5, and R6). G: Large diameter class; M: Middle diameter class; P: small diameter class. Bars 
followed by equal letters in the same diameter class are grouped by the Scott-Knott test $(\mathrm{p}<0.05)$.

Embora o manejo R6 tenha reduzido o NF (Figura 2) e a Prod T (Figura 3A), essa técnica contribuiu para o aumento da frequência relativa de frutos grande (FFG), com 86,2\%, portanto, maior do que as outras técnicas de raleio (Figura 3B). Apesar da maior FFG pela técnica R6, a Prod $G$ não se diferenciou do R3, que apresentou maior Prod T do que R6. Nas técnicas R1, R2 e R4 foi observado os menores valores de FFG, porém as frequências relativas de frutos médios (FFM) e pequenos (FFP) foram maiores do que R3, R5 e R6 (Figura 3B). R1, R2 e R4, apresentaram FFM e FFP de 39,4\% e 8,8\%, correspondente a um incremento de 0,2 e 1,9 vezes em relação aos demais raleios.

A análise de componentes principais foi capaz de sintetizar os efeitos observados, apresentando, de forma multivariada, os reflexos do raleio na produção e na qualidade morfológica de frutos de tomate (Figura 4). Nessa análise, verificou-se que as variáveis PMF, DTM, DLM e FFG foram inversamente relacionadas com o NF. Isso demonstra que o aumento em peso e tamanho dos frutos de tomate é favorecido pela redução da quantidade de frutos por planta. A Prod T foi influenciada pelo equilíbrio entre NF e Prod G, uma vez que essas variáveis apresentaram vetores opostos e mais próximos à Prod T. Com isso, observou-se a formação de dois grupos de respostas ao uso do raleio: o primeiro grupo, constituído pelas técnicas R3, R5 e R6, contribui para frutos mais pesados e de melhor qualidade morfológica; e o segundo grupo, formado pelas técnicas R1, R2 e R4, que favorece maior número de frutos, mas de menor tamanho e peso.

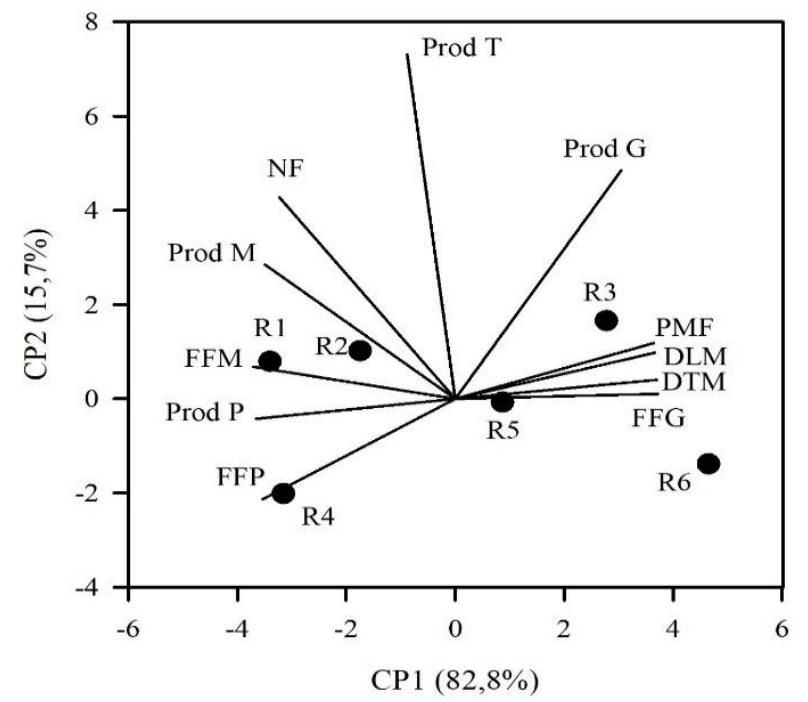

Figura 4. Análise de componentes principais (ACP) para as variáveis respostas, número de frutos por planta (NF), produção de frutos total $(\operatorname{Prod} \mathrm{T})$, na classe grande $(\operatorname{Prod} \mathrm{G})$, médio $(\operatorname{Prod} \mathrm{M})$ e pequeno $(\operatorname{Prod}$ $\mathrm{P})$, peso médio dos frutos (PMF), diâmetro transversal médio (DTM), diâmetro longitudinal médio (DLM) e frequência de frutos na classe grande (FFG), classe médio (FFM), classe pequeno (FFP), e técnicas de raleio: R1, R2, R3, R4, R5 e R6.

Figure 4. Principal component analysis (PCA) for the variables responses, number of fruits per plant (NF), total fruit production (Prod $\mathrm{T})$, in the large class (Prod L), middle (Prod M) and small (Prod S), average weight of fruits (AWF), mean transversal diameter (MTD), mean longitudinal diameter (MLD) and fruit frequency in the large class (FFL), middle class (FFM) and small class (FFS). The studied thinning techniques: R1, R2, R3, R4, R5, and R6.

\section{DISCUSSÃO}

Os resultados encontrados no presente estudo mostram o potencial de resposta ao raleio do tomateiro do grupo Santa Cruz (Figura 2, 3 e 4), modificando os parâmetros morfológicos relacionados a qualidade dos frutos. A qualidade dos tomates colhidos é baseada em suas características químicas, bioquímicas e morfológicas, e o incremento dessas variáveis geram frutos com maior valor agregado (GUIMARÃES et al., 2008; MATOS et al., 2012). O raleio de frutos é uma técnica de fácil execução e quando empregadas corretamente, aumentam a qualidade morfológica dos frutos de tomate (AZEVEDO et al., 2010; MANTUR; PATIL, 2008; PENGFEI et al., 2017; SHIRAHIGE et al., 2010).

$\mathrm{O}$ raleio aumenta a quantidade de radiação interceptada pela planta e modifica a sua relação fonte/dreno, reduzindo a competição por fotoassimilados entre frutos (BECKLES, 2012; LINS et al., 2013; MAX et al., 2016). A magnitude da variação na relação fonte/dreno também é dependente da cultivar e do grupo de tomate estudado (BECKLES, 2012). Li et al. (2015) verificaram essa dependência genotípica do tomateiro na relação fonte/dreno, que influencia linearmente o teor de carboidratos nas folhas e o tamanho dos frutos.

As técnicas de raleio adotadas no presente estudo alteraram a Prod $\mathrm{G}$ e as características morfológicas dos frutos (DLM, DTM e PMF) (Figura 1, 2 e 3). Essa mudança na qualidade morfológica do tomate está relacionada à natureza dos frutos, em que são considerados drenos metabólicos de alta exigência. A redução do número de frutos na planta modifica a relação fonte/dreno e o tamanho e peso dos frutos produzidos; contudo, esse efeito pode ser similar para diferentes técnicas de raleio (BECKLES, 2012; DUARTE; PEIL, 2010; LINS et al., 2013; MATSUDA et al., 2011; SHIRAHIGE et al., 2010), como observado entre R3 e R6 (Figura 2, 3 e 4).

Além do raleio, outras técnicas podem ser utilizadas para aumentar a qualidade morfológica de frutos de tomate, como o uso de ácido giberélico $\left(\mathrm{GA}_{3}\right)$. Ayub; Rezende (2010) estudaram a aplicação de doses de $\mathrm{GA}_{3}$ no tomate e verificaram aumentos no DTM, DLM e PMF na ordem de 6,6, 6,5 e $16,3 \%$, respectivamente, em relação ao tratamento sem $\mathrm{GA}_{3}$. Entretanto, foi observado no presente trabalho que o uso da técnica certa de raleio pode contribuir ainda mais para os atributos morfológicos do fruto, uma vez que o R3 e R6 favoreceram aumento médio de 10,1, 9,6, e 31,7\%, respectivamente, para DTM, DLM e PMF em relação ao cultivo sem raleio. Além disso, destaca-se que aplicação de $\mathrm{GA}_{3}$ apresenta algumas desvantagens, como maior custo e necessidade de mão de obra mais qualificada; já o raleio possui menor custo, maior facilidade de execução e ausência da dependência de insumos, tendo maior aceitação pelos produtores (MABOKO et al., 2011; MANTUR; PATIL, 2008; SHIRAHIGE et al., 2010).

$\mathrm{O}$ efeito positivo do raleio na melhoria da qualidade e produção de frutos de tomate, foi verificado por Shirahige et al. (2010). Esses autores conduziram plantas de tomate do grupo Santa Cruz com 8 racemos, mantendo 6 frutos por racemo, e observaram incrementos médios de 5,4, 4,3, 5,5, e $5,9 \%$, respectivamente, para a produtividade comercial, PMF, DTM e DLM. Mantur; Patil (2008) também relataram a importância do raleio para o aumento das características produtivas e morfológicas do tomate, porém sem especificar a quantidade de frutos remanescentes após o raleio.

Guimarães et al. (2008) mostraram que o aumento da qualidade dos frutos pelo raleio depende de como essa prática 
é efetuada e que determinadas técnicas de raleio podem não apresentar o efeito esperado na produção e na qualidade morfológica do fruto. Isso foi verificado no presente trabalho, por meio da adoção das práticas R2 e R4 (Figura 2 e 3). No estudo desenvolvido por Guimarães et al. (2008) não foi verificado diferenças no teor de sólidos solúveis (TSS) e totais (TST) no fruto pelo uso do raleio. Entretanto, Abdel-Razzak et al. (2016) verificaram que a redução do número de frutos por racemo, devido ao uso do raleio, aumentou o TSS, vitamina C, acidez titulável, teor total de açúcares, sendo que esses efeitos podem ser de até $17,1,7,2,9,0$ e 19,5\% respectivamente, quando são mantidos 6 frutos por racemo. $\mathrm{O}$ aumento no teor de açúcares no fruto de tomate também foi evidenciado por Pengfei et al. (2017), quando utilizaram 8 ou 13 frutos por racemo, melhorando o sabor do fruto. $\mathrm{O}$ efeito observado por esses autores está intrinsecamente relacionado ao aumento da partição de fotoassimilados das folhas para os frutos, o que melhora sua qualidade bioquímica e sensorial.

A qualidade bioquímica em função de práticas de raleio tem sido bem documentada, contudo, poucas avaliações na qualidade morfológica são relatadas, o que está diretamente relacionado ao interesse primário do produtor e do consumidor. Assim, o presente estudo mostra que a faixa de variação entre esses atributos morfológicos do fruto em função do raleio foram de 60 a $68 \mathrm{~mm}$ para DLM, 63 a $69 \mathrm{~mm}$ para DTM e 122,1 a 178,9 $\mathrm{g}_{\text {fruto }^{-1}}$ para PMF (Figura 2). Esses resultados foram semelhantes ao encontrado por Shirahige et al. (2010), que em resposta ao raleio obtiveram valores entre 56 a $65 \mathrm{~mm}, 61$ a $79 \mathrm{~mm}$ e 106,2 a 150,3 $\mathrm{g} \mathrm{fruto}^{-1}$, respectivamente, para DLM, DTM e PMF. Desse modo, constatou-se que o uso das técnicas R3 e R6 foram responsáveis pelos maiores valores de PMF, DLM e DTM (Figura 2), sendo superiores às medidas encontradas no estudo realizado por Shirahige et al. (2010).

A técnica de raleio R6 mostrou um incremento relativo na produção de frutos grandes, conforme observado pelo aumento da Prod G e da FFG (Figura 3), entretanto, devido ao menor NF e menor Prod M e Prod P, houve queda da Prod T. Essa resposta causada pela técnica R6 foi similar ao relatado por Abdel-Razzak et al. (2016), que com o raleio, mantendo 6 frutos de tomate por racemo, houve queda da Prod P e Prod M, e incremento na Prod G e FFG, contudo, a Prod T mostrou redução de 21,4\% em relação ao cultivo sem o uso do raleio.

A competição entre frutos por fotoassimilados, resulta em frutos de menor peso e tamanho (HESAMI et al., 2012; LINS et al., 2013), esta relação negativa foi observada pelo uso das técnicas R1 e R2 (Figura 2), que com o aumento do NF diminuiu o PMF. Essa relação negativa entre NF e PMF também foi relatada por Azevedo et al. (2010), que estudaram plantas de tomate com diferentes números de hastes por planta.

Com os resultados de NF também foi possível verificar que essa variável nem sempre guarda relação com a produção, uma vez que o uso da técnica R3 diminui o número de frutos e apresentou maior produção total, especialmente aqueles de maior tamanho (Figura 2 e 3). Max et al (2016) conduzindo plantas de tomate do grupo Santa Cruz com 5 frutos por racemo, também observaram que com a redução do NF houve aumento da qualidade morfológica dos frutos. No entanto, as técnicas de raleio adotadas por Max et al. (2016), diminuíram a produção total por planta.

Neste trabalho, verificou-se que a produção total de tomates submetidos a diferentes práticas de raleio está condicionada a um equilíbrio entre NF e atributos morfológicos dos frutos (PMF, DLM e DTM) (Figura 4). Isso demonstra que os frutos remanescentes do tipo de raleio correto têm maior potencial de desenvolvimento, gerando condições que propiciam maior qualidade morfológica dos frutos. Para uma mesma classe de diâmetro, frutos que atinjam maior tamanho e peso apresentam maior aceitação pelo mercado consumidor, mas essa melhoria na qualidade do fruto não deve reduzir a produtividade total (BECKLES, 2012; MABOKO et al., 2008; MATOS et al., 2012; MONTE et al., 2013). Essa condição foi alcançada neste estudo, com o uso da técnica R3 na condução do tomateiro, que manteve a Prod T similar ao R1 e, ainda aumentou Prod G, o que pode refletir em aumento de rentabilidade devido ao maior preço pago pelos frutos dessa classe.

Há carência de trabalhos na cultura do tomateiro do grupo Santa Cruz que elucidem o impacto de diferentes técnicas de raleio na produção e frequência relativa em cada classe de diâmetro de frutos produzidos, bem como na sua qualidade morfológica, concentrando a maioria dos trabalhos somente na Prod T (MANTUR; PATIL, 2008; SHIRAHIGE et al., 2010). Portanto, os resultados deste trabalho esclarecem e auxiliam na tomada de decisão quanto ao uso do raleio, relacionando aspectos produtivos e qualitativos de frutos de tomate, unindoos em classes de diâmetro. Além disso, este estudo fortalece a importância do tipo de raleio a ser empregado no tomateiro, para que alcance maior rentabilidade com a produção de tomate.

\section{CONCLUSÕES}

O cultivo de plantas de tomate com 7 racemos, com 6 frutos por racemo até o $4^{\circ}$ racemo e acima deste, mantendo 4 frutos por racemo (R3), se destaca entre as demais técnicas de raleio por aliar alta produção e frutos de diâmetro transversal e longitudinal maiores que $65 \mathrm{~mm}$.

A produção de frutos grandes diminui com técnicas de raleio que proporcionam mais de seis frutos por racemo, enquanto plantas conduzidas com menos de sete racemos reduz a produção de tomate.

\section{AGRADECIMENTOS}

Ao Instituto Federal Minas Gerais-campus Bambuí pelo apoio financeiro, espaço físico e insumos essenciais para a condução desse estudo.

\section{REFERÊNCIAS}

ABDEL, H. A; WAHB-ALLAH, M.; IBRAHIM, A.; ALENAZI, M.; ALSADON, A. et al. Response of cherry tomato to irrigation levels and fruit pruning under greenhouse conditions. Journal of Agricultural Science and Technology, Tehran, v. 18, n. 10, p. 1091-1103, out. 2016. DOI: https://dx.doi.org/10.3923/ajcs.2013.275.285

AYUB, R. A.; REZENDE, B. L. A. Contribuição do ácido giberélico no tamanho de frutos do tomateiro. Biotemas, Florianopolis, v. 23, n. 4, p. 25-28, dez. 2010. DOI: http://dx.doi.org/10.5007/2175-7925.2010v23n4p25

AZEVEDO, V. F.; ABBOUD, A. C. de S.; CARMO, M. G. F do. Row spacing and pruning regimes on organically grown cherry tomato. Horticultura Brasileira, Brasília v. 28, n. 4, p. 389-394, out./dez. 2010. DOI: https://dx.doi.org/10.1590/S0102-05362010000400002

BECKLES, D. M. Factors affecting the postharvest soluble solids and sugar content of tomato (Solanum lycopersicum 
L.) fruit. Postharvest Biology and Technology, Amsterdam, v. 63, n. 1, p. 129-140, jan. 2012. DOI: https://dx.doi.org/10.1016/j.postharvbio.2011.05.016

DUARTE, T. S.; PEIL, R. M. Relações fonte: dreno e crescimento vegetativo do meloeiro. Horticultura Brasileira, v. 28, n. 3, p. 271-276, jul./set. 2010. DOI: http://dx.doi.org/10.1590/S0102-05362010000300005

ENGELS, C.; KIRKBY, E.; WHITE, P. Mineral nutrition, yield and source-sink relationships. In: MARSCHNER, P. Marschner's mineral nutrition of higher plants (Third Edition). Cambridge: Elsevier/Academic Press, 2012, p. 85-133.

FERREIRA, E. B.; CAVALCANTI, P. P. NOGUEIRA, D. A. ExpDes.pt: Experimental Designs pacakge (Portuguese). R package version 1.1.2. 2013. Disponível em: <http://CRAN.R-project.org/package=ExpDes.pt.>. Acesso em: 30 Jan 2018.

FILGUEIRA, F. A. R.; OBEID, P. C.; MORAIS, H. J.; SANTOS, W. V.; BARBOSA, V. Tomate. In: RIBEIRO, A. C.; GUIMARÃES, P. T. G; ALVAREZ V., V. H. Recomendação para o uso de corretivos e fertilizantes em Minas Gerais, $5^{a}$ Aproximação. Viçosa: Comissão de Fertilidade do Solo do Estado de Minas Gerais, 1999. p. 207-208.

GUIMARÃES, M. A.; SILVA, D. J. H.; FONTES, P. C. R.; MATTEDI, A. P. Produtividade e sabor dos frutos de tomate do grupo salada em função de podas. Bioscience Journal, Uberlandia, v. 24, n. 1, p. 32-38, jan./mar. 2008.

HESAMI, A.; KHORAMI, S. S.; HOSSEINI, S. S. Effect of shoot pruning and flower thinning on quality and quantity of semi-determinate tomato (Lycopersicon esculentum Mill.). Notulae Scientia Biologicae, Cluj-Napoca, v. 4, n. 1, p. 108-11, jan. 2012. DOI: https://dx.doi.org/10.15835/nsb417179

LI, T.; HEUVELINK, E.; MARCELLIS, L. F. M. Quantifying the source sink balance and carbohydrate contente in three tomato cultivars. Frontiers in Plant Science, v. 6, n. 416, p. 1-10, jun. 2015. DOI: https://dx.doi.org/10.3389/fpls.2015.00416

LINS, H. A.; QUEIROGA, R. C. F.; PEREIRA, A. M.; SILVA, G. D.; ALBUQUERQUE, J. R. T. Produtividade e qualidade de frutos de melancia em função de alterações na relação fonte-dreno. Revista Verde, Mossoró, v.8, n.3, p. 143-149, jul./set. 2013.

MABOKO, M. M.; PLOOY, C. P.; CHILOANE, S. Effect of plant population, fruit and stem pruning on yield and quality of hydroponically grown tomato. African Journal of Agricultural Research, v.6, n.22, p.5144-5148, 2011.

MANTUR, S. M.; PATIL, S. R. Influence of spacing and pruning on yield of tomato grown under shade house. Karnataka Journal of Agricultural Sciences, Dharwad, v. 21, n. 1, p. 97-98, jan./mar. 2008.

MAPA_MINISTÉRIO DA AGRICULTURA, PECUÁRIA E ABASTECIMENTO. Norma de identidade, qualidade, acondicionamento, embalagem e apresentação do tomate. 1995 . Disponível em: $<$ http://sistemasweb.agricultura.gov.br/sislegis/action/det alhaAto.do?method=visualizarAtoPortalMapa\&chave $=19$ 20192566>. Acesso em: 30 Jan 2018.

MATOS, E. S.; SHIRAHIGE, F. H.; MELO, P. C. T. de. Desempenho de híbridos de tomate de crescimento indeterminado em função de sistemas de condução de plantas. Horticultura Brasileira, Brasília, v. 30, n. 2, p.
240-245, abr./jun. $2012 . \quad$ DOI: http://dx.doi.org/10.1590/S0102-05362012000200010

MATSUDA, R.; SUZUKI, K.; NAKANO, A.; HIGASHIDE, T.; TAKAICHI, M. Responses of leaf photosynthesis and plant growth to altered source-sink balance in a Japanese and a Dutch tomato cultivar. Scientia Horticulturae, Amsterdam, v. 127, n. 4, p. 520-527, fev. 2011. DOI: https://dx.doi.org/10.1016/j.scienta.2010.12.008

MAX, J. F. J.; SCHMIDT, L.; MUTWIWA, U. N.; KAHLEN, $\mathrm{K}$. Effects of shoot pruning and inflorescence thinning on plant growth, yield and fruit quality of greenhouse tomatoes in a tropical climate. Journal of Agriculture and Rural Development in the Tropics and Subtropics (JARTS), Leipzig, v.117, n. 1, p. 45-56, jan./mar. 2016.

MONTE, J. A.; CARVALHO, D. F.; MEDICI, L. O.; SILVA, L. D. B.; PIMENTEL, C. Growth analysis and yield of tomato crop under different irrigation depths. Revista Brasileira de Engenharia Agrícola e Ambiental, Jaboticabal, v. 17, n. 9, p. 926-931, set. 2013. DOI: http://dx.doi.org/10.1590/S1415-43662013000900003

OKSANEN, J.; BLANCHET, F. G.; FRIENDLY, M.; KINDT, R.; LEGENDRE, P.; MCGLINN, D.; MINCHIN, P. R.; O'HARA, R. B.; SIMPSON, G. L.; SOLYMOS, P.; STEVENS, M. H. H.; SZOECS, E.; WAGNER, H. Vegan:Community Ecology Package. $R$ package version 2.4-3. 2017. Disponível em: <https://CRAN.Rproject.org/package=vegan $>$. Acesso em: 30 Jan 2018.

PENGFEI, Z.; YANYAN, D.; MASATERU, S.; NATSUMI, M.; KENGO, I. Interactions of Salinity Stress and Flower Thinning on Tomato Growth, Yield, and Water Use Efficiency. Communications in Soil Science and Plant Analysis, New York, v. 48, n. 22, p. 2601-2611, 2017. DOI: https://dx.doi.org/10.1080/00103624.2017.1411508

R CORE TEAM. R: A language and environment for statistical computing. 2018. Disponível em: <https://www.R-project.org/>. Acesso em: 30 Jan 2018.

RODRÍGUEZ-MENDOZA, M. N.; BACA-CASTILLO, G.; GARCÍA-CUÉ, J. L.; URRIETA-VELÁZQUEZ, J. A. Aclareo de frutos y aspersiones foliares de calcio y miel de abeja sobre la calidad de tomate tipo costilla. Revista Fitotecnia Mexicana, v. 38, n. 2, p.197-204, 2015.

SCHMIDT, J.; MENEGHELLI, C. M.; KRAUSE, M. R.; HELL, M. V.; OLIVEIRA, M. L.; COLOMBO, J. N.; HADDADE, I. R. Desempenho do tomateiro em sistema de tutoramento vertical em espiral conduzido com uma ou duas hastes. Nativa, Sinop, v. 6, n. 4, p. 338-344, jul./ago. 2018. DOI: http://dx.doi.org/10.31413/nativa.v6i4.5443

SHIRAHIGE, F. H.; MELO, A. M. T.; PURQUERIO, L. F. V.; CARVALHO, C. R. L.; MELO, P. C. T. Produtividade e qualidade de tomates Santa Cruz e Italiano em função do raleio de frutos. Horticultura Brasileira, Brasília, v. 28, n. $3, \quad$ p. 292-298, jul./set. 2010. http://dx.doi.org/10.1590/S0102-05362010000300009

SILVA, P. A.; RABELO, J. S.; GUIMARÃES, M. A.; SILVA, J. C. V.; OLIVEIRA, L. S. C. Sistemas de condução na produção comercial de tomate "cereja". Nativa, Sinop, v. 5, n. 5, p. 316-319, set./out. 2017. DOI: http://dx.doi.org/10.5935/2318-7670.v05n05a03

SOWLEY, E. N. K.; DAMBA, Y. Influence of staking and pruning on growth and yield of tomato in the Guinea Savannah Zone of Ghana. International Journal of Scientific and Technology Research, Paris, v. 2, n. 12, p. 103-108, dez. 2013. 